Postprint: Hou T., Meerbergen K., Roels S., Janssen H., 2020. POD-DEIM model order reduction for nonlinear heat and moisture transfer in building materials, Journal of Building Performance Simulation, 13:6, 645-661, DOI: 10.1080/19401493.2020.1810322

\title{
POD-DEIM model order reduction for nonlinear heat and moisture transfer in building materials
}

\author{
Tianfeng $\mathrm{Hou}^{1 *}$, Karl Meerbergen ${ }^{2}$, Staf Roels ${ }^{1}$, Hans Janssen ${ }^{1}$
}

${ }^{1}$ KU Leuven, Department of Civil Engineering, Building Physics Section, Kasteelpark Arenberg 40, 3001 Heverlee, Belgium.

${ }^{2} \mathrm{KU}$ Leuven, Department of Computer Science, Numerical Analysis and Applied Mathematics section, Celestijnenlaan 200a, 3001Heverlee, Belgium.

\section{Abstract}

In this paper, the proper orthogonal decomposition (POD) method and the discrete empirical interpolation method (DEIM) are combined to construct a reduced order model for a cost optimal simulation of heat and moisture transfer in building materials. The POD-DEIM performance is assessed via two applications: a relatively simple case study on nonlinear heat conduction and a more complex case study on nonlinear moisture redistribution. In both, the results of the PODDEIM model are compared to results from POD and finite volume methods simulations, to evaluate the accuracy of the POD-DEIM as a function of the numbers of construction modes and interpolation points. Also, as the number of POD construction modes and DEIM interpolation points does not entirely represent the computational cost of different models, the accuracies of the different models are compared as a function of the respective calculation times, to provide a fair comparison of their computational performances. Further, the use of POD-DEIM to simulate a problem different from the POD training snapshot simulation is investigated. The outcomes show that when a rather highly accurate result is desired, POD-DEIM model is capable of reducing the computational cost relative to POD and FVM.

Keywords: Proper orthogonal decomposition; Discrete empirical interpolation method; Finite volume method; Heat and moisture transfer; Model order reduction; Building hygrothermal performance

* Corresponding author. Tel:: +32 16328832; fax: + 3216321980

E-mail address: tianfeng.hou@kuleuven.be 


\section{Introduction}

The use of numerical simulations for modelling heat and moisture transport problems is common, given that these allow a multitude of scenarios and factors to be evaluated smoothly. In building physics, hygrothermal models are often used to simulate the heat, air and moisture transfer in oneor multi-dimensional configurations: in literature we can find many computer-based tools for building hygrothermal performance prediction, usually based on the standard methods for space and time discretization, such as Match, WUFI, Delphin or Hamfem [1]-[4]. These standard methods for space and time discretization can be very time consuming though, due to the strongly nonlinear equations, the complex multi-dimensional spatial domains, and/or the lengthy simulation intervals required. To lessen the computational expense, a faster surrogate model, which strongly reduces the calculation time of hygrothermal simulation, is desirable.

Proper orthogonal decomposition (POD) has the potential to reduce the computational complexity and computational expense for simulating a large complex system, by approximating the high dimensional process by its "most relevant information". POD has been successfully applied in a variety of engineering fields, such as image processing, signal analysis, data compression, and lately equally in building physics [5]-[8]. Recently, Hou et al. [9] employed POD to reduce the calculation time for simulating linear heat conduction in the frame of building thermal performance assessment. It was demonstrated that the POD model outperforms the conventional finite element model for simulating these linear heat conduction problems, and that the use of POD to simulate a problem different from the training snapshot simulation can provide an accurate result. Hence the robustness of the POD method is confirmed. However, for nonlinear problems, such as the highly nonlinear heat and moisture transfer in building materials, POD suffers from the same shortcomings as conventional simulation methods. As in the finite element method (FEM) and the finite volume method (FVM), the inefficiency of POD arises from the high computational expense of repeatedly evaluating the nonlinear terms still in the scope of the dimensions of the original problem [10]. To overcome this bottleneck, Chaturantabut et al. [10] proposed the discrete empirical interpolation method (DEIM). The basic idea of DEIM is to approximate these nonlinear terms by combining projection with interpolation. DEIM projects the nonlinear terms onto a reduced basis and evaluates these terms at specific locations only, which are selected by a greedy algorithm. The component values at other locations are interpolated through the reduced basis by an interpolation matrix that is pre-computed offline [11].

POD-DEIM has been successfully employed in many fields [12]-[15], and its potential to reduce the computational expense of nonlinear simulations has been addressed by Chaturantabut et al. [10]. However, to the best of the authors' knowledge, there is no prior research in the field of building physical engineering giving a detailed investigation of the feasibility of POD-DEIM for modelling the non-linear heat and moisture transfer in building components. Moreover, since the computational complexity does not fully cover the computational expense, the accuracies of different approaches need to be compared as a function of their calculation time, to provide a fairer illustration of the performance of POD-DEIM versus the conventional methods such as FVM. In addition, since the POD basis is constructed by the earlier results from a single simulation with the original model, a rigorous analysis of the robustness and flexibility of POD-DEIM for simulating 
problems different from the original configuration is crucial, and should be illustrated extensively. This paper targets these three research questions by applying POD-DEIM on two applications: an a relatively simple case study of nonlinear heat conduction through a material and a rather more complex case study on moisture redistribution in a material. This paper is built up as follows. In section 2, a brief introduction of POD and POD-DEIM is given, focusing on their potential use for simulating nonlinear heat and moisture transfer in building materials. In section 3 and 4, the two illustrative case studies are introduced, and the performance of FVM, POD and POD-DEIM are compared based on their accuracy and expense. Finally, in section 5 our conclusions on the main findings on the potential of POD-DEIM are put forward.

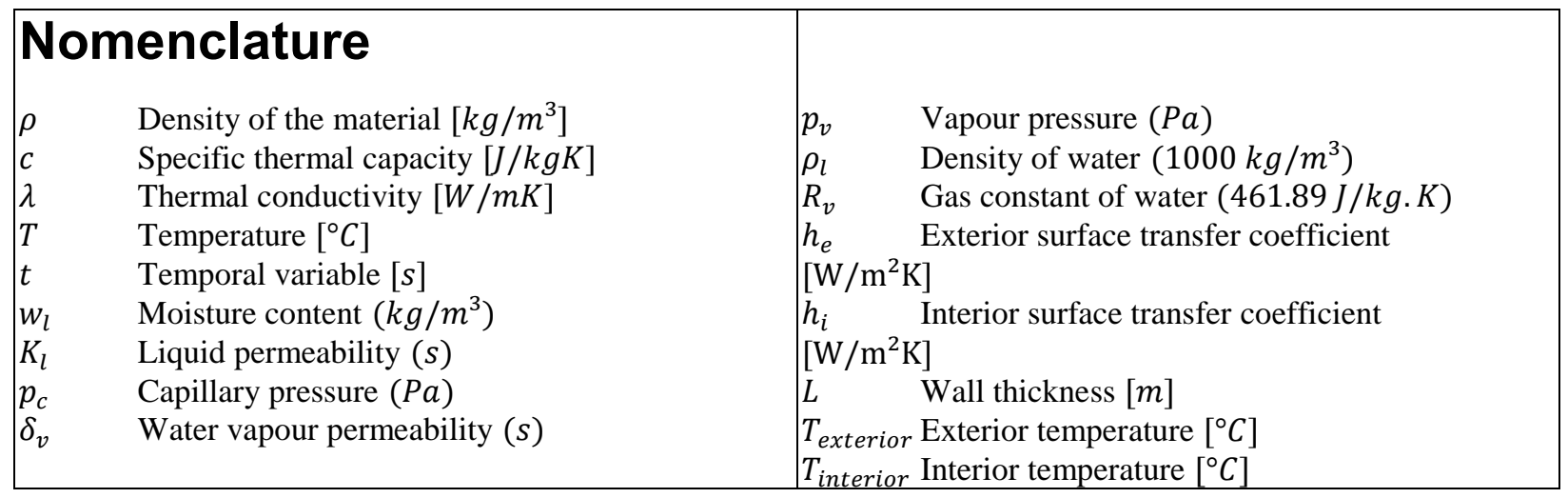

\section{POD and DEIM for modelling heat and moisture transfer}

The simulation of heat and moisture transfer in building materials is to get numerical simulation results for the heat and moisture transfer equation, combined with information on the component geometry, material properties and boundary conditions. The conventional simulation models are typically based on numerical methods applying a space and a time discretization, for instance, the FVM. It should be mentioned that, the two illustrative case studies in this paper are respectively focusing on heat transfer and moisture transfer, no coupled heat and moisture transfer phenomena are hence involved. Heat and moisture transfer in building materials can be respectively modelled by the heat and moisture transfer equations (1) and (2) [4].

$$
\begin{aligned}
& \rho c \frac{\partial T}{\partial t}=\nabla(\lambda \nabla \mathrm{T}) \\
& \frac{\partial w_{l}}{\partial t}=\nabla\left(K_{l} \nabla p_{c}\right)+\nabla\left(\frac{\delta_{v} p_{v}}{\rho_{l} R_{v} T} \nabla p_{c}\right)
\end{aligned}
$$

All symbols appearing in equations 1,2 are clarified in the nomenclature section at the start of this paper. The standard FVM for spatial discretization together with the common implicit Euler for temporal discretization to numerically solve the heat or moisture transfer equations yields a set of algebraic equations [4]: 


$$
\begin{aligned}
& \left(C^{t+\Delta t, p}+\Delta t K^{t+\Delta t, p}\right) u^{t+\Delta t, p+1}=F^{t+\Delta t, p} \Delta t+C^{t+\Delta t, p} u^{t+\Delta t, p}-\Delta t K^{t+\Delta t, p} u^{t+\Delta t, p}- \\
& \left(S^{t+\Delta t, p}-S^{t}\right)
\end{aligned}
$$

where $p$ indicates the iteration number, $u$ is the vector of unknown temperatures or capillary pressures at the spatial points, $K$ denotes the stiffness matrix, $C$ represents the mass matrix, $F$ is the load vector and $S$ are the conservation terms in relation to the energy or mass. Additionally, $t$ and $\Delta t$ respectively stand for the current time and the length of time step. The resulting system of algebraic equations is nonlinear as the mass, stiffness and load matrices all depend on the unknown values of temperature or capillary pressure and hence a Newton-Raphson iterative scheme is implemented and the final resulting system of equations to be solved is [4]:

$$
\begin{aligned}
& \left(C^{t+\Delta t, p}+\Delta t K^{t+\Delta t, p}-\Delta t\left(\frac{d F^{t+\Delta t, p}}{d u}\right)\right) \Delta u^{t+\Delta t, p+1}=F^{t+\Delta t, p} \Delta t-\Delta t K^{t+\Delta t, p} u^{t+\Delta t, p}- \\
& \left(S^{t+\Delta t, p}-S^{t}\right)
\end{aligned}
$$

\subsection{POD}

The introduction of proper orthogonal decomposition has been shortly presented by Hou et al. [9], with an application in the field of linear heat conduction through building materials. In this section we repeat the basic principles of POD and then extend its content and scope to the simulation of nonlinear heat and moisture transfer. The POD method was first proposed by Kosambi in 1943 [16], and is also known as Karhunen-Loeve decomposition, principal component analysis, or singular value decomposition, and the connections of the methods are provided by Liang et al. [17]. The key idea of POD is condensing a high-dimensional process to its 'most relevant information' only. In this paper, that 'most relevant information' is extracted from earlier simulation results of the original numerical model through singular value decomposition (SVD) [18]. More specifically, the earlier simulation results are a set of data $u(x, t) \in R_{n \times m}$ containing all the results for all $n$ spatial points $x_{i}$ and all $m$ temporal points $t_{j}$, which is called the matrix of snapshots. Through SVD the matrix of snapshots is decomposed into three distinct matrices, $u=$ $B \sum V$, with $\mathrm{B} \in R_{n \times n}, \sum \in R_{n \times m}$ and $V \in R_{m \times m}$, as shown in Figure 1 . Here, $B$ contains the set of orthonormal eigenvectors of $u u^{\mathrm{T}}$ and is called the matrix of construction modes, while $\sum$ is a rectangular diagonal matrix containing the singular values of $u$ in a reducing order on its main diagonal. Next, for the purpose of optimality only $k$ POD modes (with $k<n$ ) are selected and the snapshots matrix $u(x, t)$ is approximated with $\underline{B}_{n \times k} \underline{\Sigma}_{k \times k} \underline{\mathrm{V}}_{k \times m}^{T}$ wherein $\underline{B}_{n \times k}, \underline{\Sigma}_{k \times k}$, and $\underline{\mathrm{V}}_{m \times k}$ are respectively the truncated versions of $B, \Sigma$ and $\mathrm{V}$, as shown in Figure 2. The truncated matrix of construction modes $\underline{B}_{n \times k}$ is used to construct the reduced model for simulating new problems. As already mentioned in many publications [19]-[21], the total energy of the physical field is equal to the sum of all the singular values of the snapshot, whereas the energy of the selected $k$ POD basis is equal to the sum of the first $k$ singular values. Therefore, the ratio of these two sums of singular values can be used as an performance indicator of the truncated POD basis. Moreover, the decay speed of the singular values indicates the degree of the interrelation among the snapshots. Normally, a fast decay rate of the singular values suggests a strong interrelation among the snapshots, and therefore a rather small number of POD basis is sufficient to provide an accurate result. 


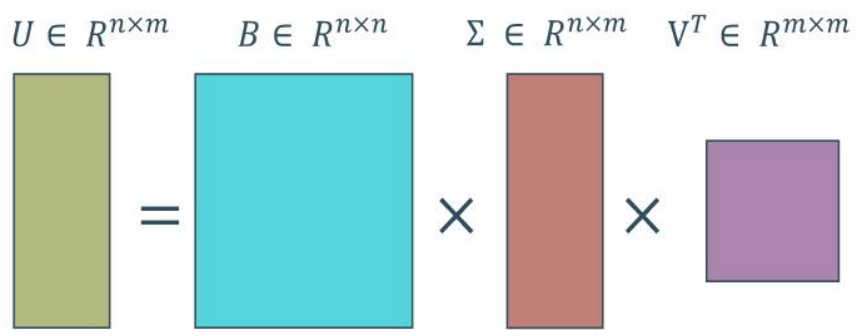

Figure 1: Graphical concept of singular value decomposition.

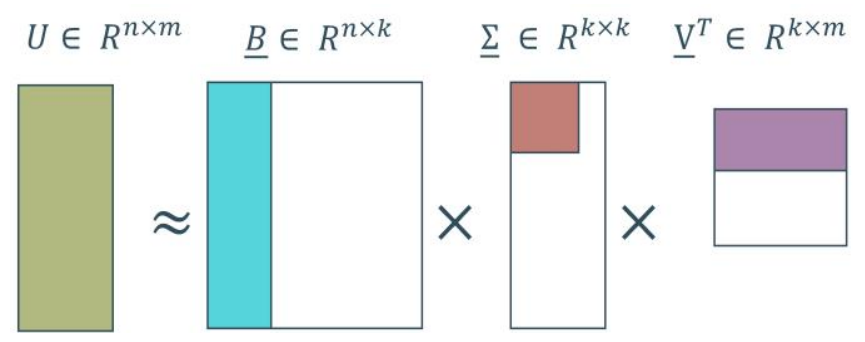

Figure 2: Truncation matrices of $B, \sum$ and $V$.

Because $u$ is approximated by $\underline{B}_{n \times k} \underline{\Sigma}_{k \times k} \underline{\mathrm{V}}_{k \times m}^{T}$, we note $\hat{\mathrm{u}}=\underline{\Sigma}_{k \times k} \underline{\mathrm{V}}_{k \times m}^{T}$, and thus $u \approx \underline{B}_{n \times k} \hat{\mathrm{u}}$. Then, by left multiplication of $\underline{B}_{k \times n}^{T}$ to (4), a reduced model is derived as follows:

$$
\begin{aligned}
& \left(\underline{\mathrm{B}}_{k \times n}^{T} C^{t+\Delta t, p} \underline{B}_{n \times k}+\Delta t \underline{\mathrm{B}}_{k \times n}^{T} K^{t+\Delta t, p} \underline{B}_{n \times k}-\Delta t \underline{\mathrm{B}}_{k \times n}^{T}\left(\frac{d F^{t+\Delta t, p}}{d u}\right) \underline{B}_{n \times k}\right) \Delta \hat{\mathrm{u}}^{t+\Delta t, p+1}= \\
& \underline{\mathrm{B}}_{k \times n}^{T} F^{t+\Delta t, p} \Delta t-\Delta t \underline{\mathrm{B}}_{k \times n}^{T} K^{t+\Delta t, p} u^{t+\Delta t, p}-\underline{\mathrm{B}}_{k \times n}^{T}\left(S^{t+\Delta t, p}-S^{t}\right)
\end{aligned}
$$

As a result, the new system (5) together with the relation $u \approx \underline{B}_{n \times k}$ û allows to approximate the solution at a reduced computational expense. Normally, the lower the number of modes selected to build the reduced model, the lower the number of degrees of freedom contained in the reduced system, which hence lowers computational expense. However, as mentioned in section 2.1, due to the nonlinearity of mass, stiffness and load matrices, in each iteration step these nonlinear matrices need to be evaluated based on the updated values of the capillary pressures and temperatures. To overcome this bottleneck and thus further reduce the computational expense, the DEIM technique is proposed.

\subsection{DEIM}

The main idea of the discrete empirical interpolation method is to approximate each nonlinear term by combining projection with interpolation. More specifically, DEIM projects the nonlinear terms onto a reduced basis and evaluates them only at specific locations, which are selected by a greedy algorithm. The component values at other locations are interpolated through the reduced basis by an interpolation matrix that can be pre-computed offline [11]. Therein, the reduced basis related to the nonlinear terms is obtained by the SVD method on their snapshots. A brief introduction of POD-DEIM is provided in the following. For the purpose of simplification, we denote the right hand side of equation (4): $F^{t+\Delta t, p} \Delta t-\Delta t K^{t+\Delta t, p} u^{t+\Delta t, p}-\left(S^{t+\Delta t, p}-S^{t}\right)$ simply by $b$. 
To reduce the computational cost of evaluating the nonlinear terms in each iteration step, we approximate these nonlinear components by projecting them onto a subspace. Suppose $b$ can be projected on a subspace of dimension $m$ spanned by the basis $D=\left[d_{1}, \ldots, d_{m}\right] \in R_{n \times m}$ as:

$$
b \approx D c
$$

where $c \in R_{m \times 1}$ is the coefficient vector of the reduced basis. Since $n>m$ the system (6) is overdetermined. To determine $c$, we select $m$ distinct rows from (6) by multiplying a predetermined transpose of a boolean matrix $P \in R_{n \times m}$ :

$$
\mathrm{P}^{T} b=\mathrm{P}^{T} D c
$$

By left multiplication of $\left(\mathrm{P}^{T} D\right)^{-1}$ to both sides of equation (7), the coefficient vector $\mathrm{c}$ can be derived:

$$
\mathrm{c}=\left(\mathrm{P}^{T} D\right)^{-1} \mathrm{P}^{T} b
$$

and therefore (6), the approximation of $\mathrm{b}$ becomes:

$$
b \approx D\left(\mathrm{P}^{T} D\right)^{-1} \mathrm{P}^{T} b
$$

By left multiplication of $D\left(\mathrm{P}^{T} D\right)^{-1} \mathrm{P}^{T}$ to both sides of equation (4) we obtain:

$$
\begin{aligned}
& D\left(\mathrm{P}^{T} D\right)^{-1} \mathrm{P}^{T}\left(C^{t+\Delta t, p}+\Delta t K^{t+\Delta t, p}-\Delta t\left(\frac{d F^{t+\Delta t, p}}{d u}\right)\right) \Delta u^{t+\Delta t, p+1}= \\
& D\left(\mathrm{P}^{T} D\right)^{-1} \mathrm{P}^{T}\left(F^{t+\Delta t, p} \Delta t-\Delta t K^{t+\Delta t, p} u^{t+\Delta t, p}-\left(S^{t+\Delta t, p}-S^{t}\right)\right)
\end{aligned}
$$

Combined with the POD method, the POD-DEIM model reduction method transfers equation (5) to

$$
\begin{aligned}
& D\left(\mathrm{P}^{T} D\right)^{-1} \mathrm{P}^{T}\left(\underline{\mathrm{B}}_{k \times n}^{T} C^{t+\Delta t, p} \underline{B}_{n \times k}+\Delta t \underline{\mathrm{B}}_{k \times n}^{T} K^{t+\Delta t, p} \underline{B}_{n \times k}-\right. \\
& \left.\Delta t \underline{\mathrm{B}}_{k \times n}^{T}\left(\frac{d F^{t+\Delta t, p}}{d u}\right) \underline{B}_{n \times k}\right) \Delta \hat{\mathrm{u}}^{t+\Delta t, p+1}=D\left(\mathrm{P}^{T} D\right)^{-1} \mathrm{P}^{T}\left(\underline{\mathrm{B}}_{k \times n}^{T} F^{t+\Delta t, p} \Delta t-\right. \\
& \left.\Delta t \underline{\mathrm{B}}_{k \times n}^{T} K^{t+\Delta t, p} u^{t+\Delta t, p}-\underline{\mathrm{B}}_{k \times n}^{T}\left(S^{t+\Delta t, p}-S^{t}\right)\right)
\end{aligned}
$$

The transpose of boolean matrix $\mathrm{P}^{T}$ guarantees that each nonlinear term of system (10) only needs to be evaluated at specific locations, while other component values are interpolated by $D\left(\mathrm{P}^{T} D\right)^{-1}$ that can be pre-computed offline. In addition, the projection basis $D$ is calculated by applying SVD on the nonlinear snapshots of $b$, and the DEIM indices which are the entries of the boolean matrix $P$ are derived from the DEIM algorithm proposed by Chaturantabut et al. [10].

\section{Case study 1: nonlinear heat transfer}

In this section, the one-dimensional thermal behavior of the wall is taken as an exemplary "calculation object" and a set of analyses are performed to provide an exhaustive evaluation of the performances of different models. To do so, the thermal behavior of a building component is simulated with the FVM, POD and POD-DEIM, wherein the conductive heat transfer equation (equation (1)) is solved under the below interior and exterior boundary conditions: 


$$
\begin{gathered}
\lambda \frac{\partial T}{\partial x}=h_{e}\left(T(0)-T_{\text {exterior }}\right) \\
-\lambda \frac{\partial T}{\partial x}=h_{i}\left(T(L)-T_{\text {interior }}\right)
\end{gathered}
$$

All symbols appearing in equations (12) and (13) are clarified in the nomenclature section at the start of this paper. A sketch of the material with boundary conditions is illustrated in Figure 3 . The simulation period is one year. All solvers necessary for the purpose have been developed by the first author. The accuracy of the obtained solutions is then evaluated by comparison with a reference solution, a FVM calculation applying 200 spatial mesh elements and a fixed time step of one hour, in order to keep the discretization errors sufficiently small. It should be mentioned that, our FVM implementation has been verified by comparison with the HAMSTAD benchmarks [22]. In order to compare the overall behavior of different models, the deviation of solutions of different models relative to the reference solution are quantified with the average absolute temperature error:

$$
\frac{\substack{1 \leq i \leq N_{x} \\ 1<j<N_{t}}}{N_{x} \times N_{t}}
$$

Here, $T_{F V M}$ and $T_{\text {reduced }}$ are respectively the temperature results of the reference solution and the reduced model with $N_{x}$ and $N_{t}$ the number of spatial and temporal discretization points of different methods.

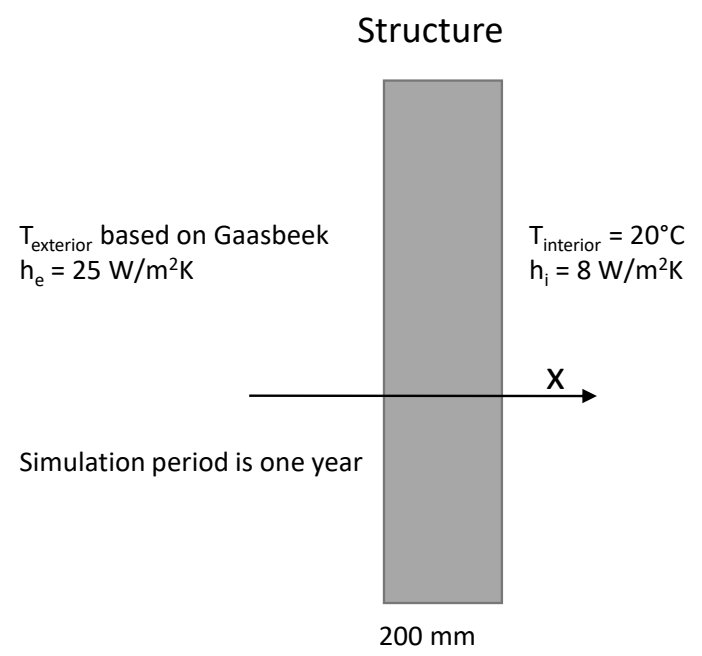

Figure 3: A sketch of the material with boundary conditions of the heat transfer case study. 


\subsection{Scenario 1: temperature-dependent thermal capacity}

As stated in section 2, to construct POD and POD-DEIM models, a snapshot from a detailed simulation is required to serve as the basis for the reduced models. In addition, the reduced model obtained by POD and POD-DEIM are usually used for simulating problems with different but comparable boundary conditions, material properties, component geometry and/or for longer simulation periods. Therefore, in this section, three research issues are studied: 1 . the accuracy and computational expense of FVM, POD and POD-DEIM constructed with the full set of snapshots, 2. the performance of POD-DEIM for simulating problems with longer simulation intervals, 3 . the accuracy of POD-DEIM for simulating problems deviating from the training snapshot simulation. The results are respectively put forward in sections 3.1.1, 3.1.2 and 3.1.3.

\subsubsection{FVM, POD, POD-DEIM constructed by the full set of solution snapshots}

For the confrontational case study of FVM, POD and POD-DEIM constructed by the full set of solution snapshots, the needed input parameters to equations (1) and (12-13) are addressed in the following. It should be mentioned that, except for the nonlinear thermal capacity, all the parameter values are kept identical to the case study presented in [9]. In relation to the component geometry, the thickness of the single building material $L$ is $0.2 \mathrm{~m}$. For the material properties, density $\rho$, and conductivity $\lambda$ of the building material are $2100 \mathrm{~kg} / \mathrm{m}^{3}$ and $1 \mathrm{~W} / \mathrm{mK}$. The thermal capacity $c$ is assumed to be temperature-dependent, being $500 \mathrm{~J} / \mathrm{kgK}$ for temperatures below $0^{\circ} \mathrm{C}$ versus 1500 $\mathrm{J} / \mathrm{kgK}$ for temperatures above $30^{\circ} \mathrm{C}$, with a linear dependence on temperature between these two bounds. The boundary conditions are restricted to combined convection and radiation, governed by (equivalent) air temperatures. The outdoor air temperature $T_{\text {exterior }}$ is taken from climate data for Gaasbeek (Belgium), while the indoor air temperature $T_{\text {interior }}$ is kept at $20{ }^{\circ} \mathrm{C}$. The initial temperature is kept at $10{ }^{\circ} \mathrm{C}$. The interior and exterior surface transfer coefficients are $h_{i}=$ $8 \mathrm{~W} / \mathrm{m}^{2} \mathrm{~K}$ and $h_{e}=25 \mathrm{~W} / \mathrm{m}^{2} \mathrm{~K}$. In this case, the calculation accuracy and computational expense of POD and POD-DEIM (constructed by the full set of snapshots from the reference FVM solution with 200 nodes) as well as FVM with "coarse discretizations" are judged. The average temperature differences (equation 14) requires the coarse-discretization FV models to have the temperature values at all the spatial points of the reference solution. To do so the intermediate values of the coarse-discretization FV models are calculated by linear interpolation.

As already mentioned in section 2.1 and 2.2, both POD and DEIM employ SVD method to obtain the POD modes and the reduced basis of the nonlinear terms. Moreover, the amount of the "most important information" contained in these POD modes and reduced basis can be reflected in the decay speed of these singular values. Normally, the fast decay of the singular values implies a high interrelation of the snapshots, and then a smaller number of construction modes is required to build a reduced model. Therefore, the decay progress of the first 45 singular values of the snapshots for both the reference solution and the nonlinear terms are shown in Figure 4. Besides, to get a more direct view of the performance of FVM, POD and POD-DEIM, both the spatial temperature profile through the building material at noon of June 30, and the temporal evolution of the temperature at $0.19 \mathrm{~m}$ of the building component during the month of June are selected as illustrative target outputs. The results are respectively presented in Figure 5 and 6, and the profile is simulated by the three models with both 2 and 16 nodes/modes. Further, to judge the accuracy of different models for the entire year, the average absolute temperature differences, see equation (14), are 
depicted in Figure 7 as a function of the number of nodes/modes. Here, all the models are constructed with 2 to 16 modes/nodes. Finally, because the number of modes/nodes do not entirely represent the computational expense of different models, the average absolute temperature differences are again presented in Figure 8, but now as a function of the calculation time, to provide a fair comparison of the computational performance of different approaches. Similar as Figure 7 , all the models are used to predict the temperature profiles for the entire year. Here, the POD and POD-DEIM models are constructed by 2 to 16 modes. To make the computational time of the FVM models as closer as the POD and POD-DEIM, the FVM models are constructed by 2 to 72 nodes, with an interval of 5 nodes. It should be mentioned that, to obtain a rather accurate approximation of the nonlinear terms all the POD-DEIM models are built by 35 DEIM points, see Figure 4. In addition, we have noticed that with more than 25 DEIM points, the accuracies of the reduced models do not increase significantly. On the other hand when the number of DEIM points is relatively low (less than 20), the simulation could be stopped due to some instability issues.

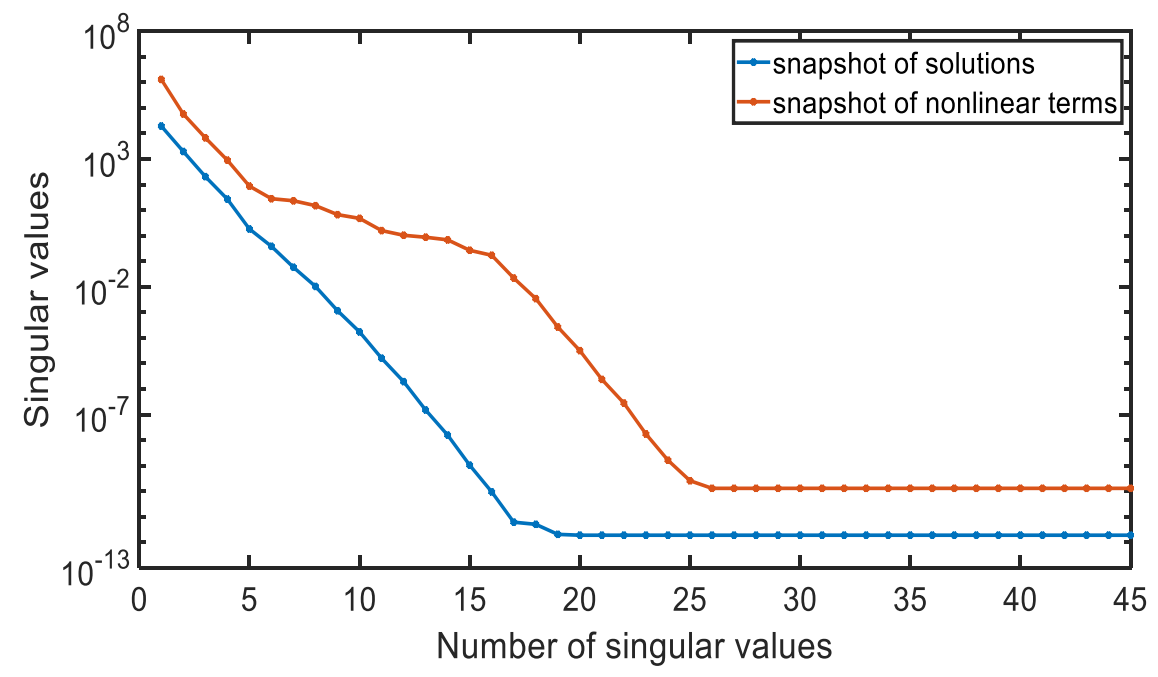

Figure 4: The decay progress of the first 45 singular values of the snapshots related to the solution and the nonlinear terms. 


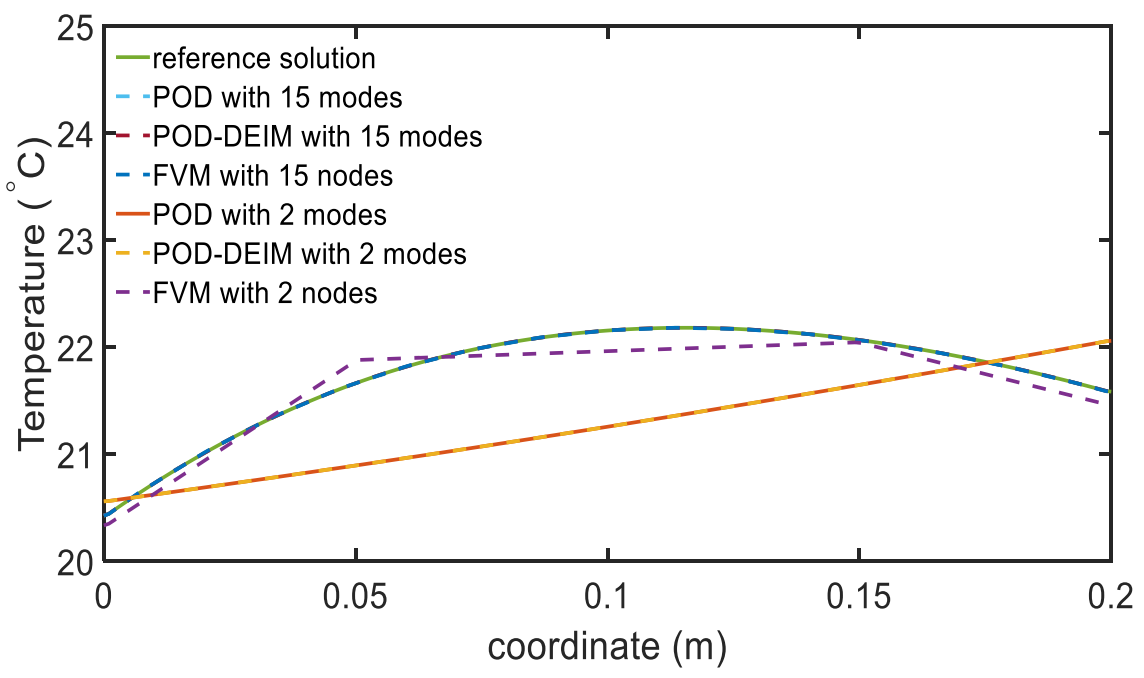

Figure 5: Spatial temperature profiles at noon of June 30, for the reference solution (solid lines) and solutions of the FVM, POD and POD-DEIM models constructed by different number of nodes/modes (dashed lines); coordinate ' $0 \mathrm{~m}$ ' refers to the exterior surface.

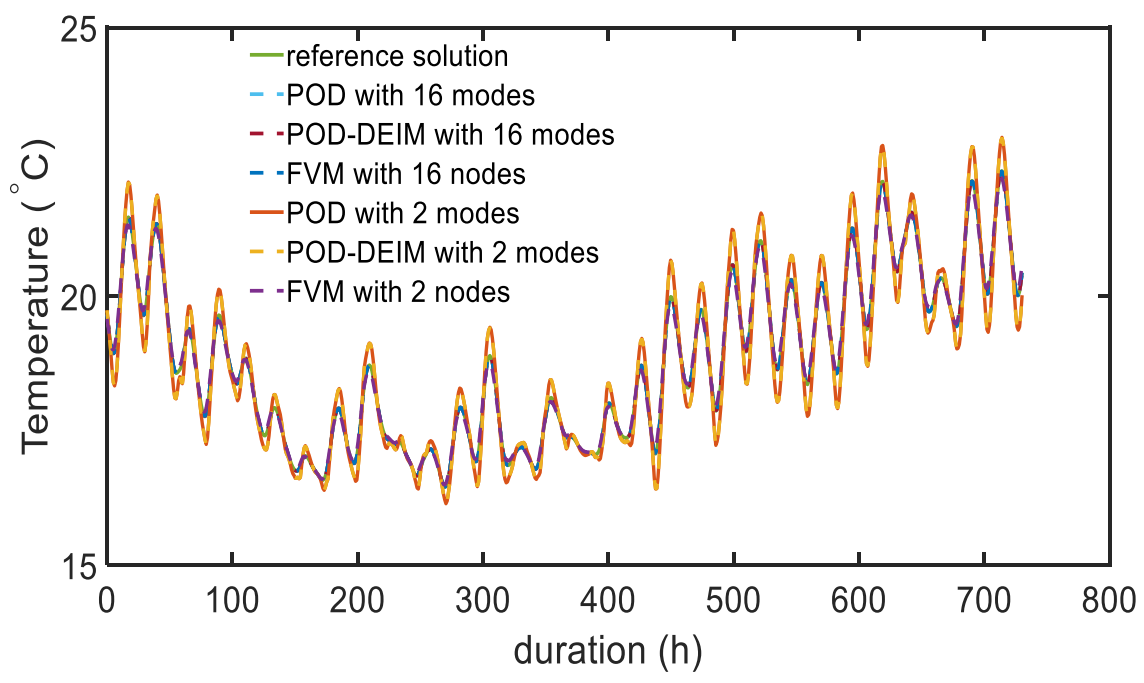

Figure 6: Temporal evolution of the temperature at $0.19 \mathrm{~m}$ of the building component during the month of June, for the reference solution (solid lines) and solutions of the FVM, POD and PODDEIM models constructed by different number of nodes/modes (dashed lines); duration ' 0 h' refers to the start of June. 


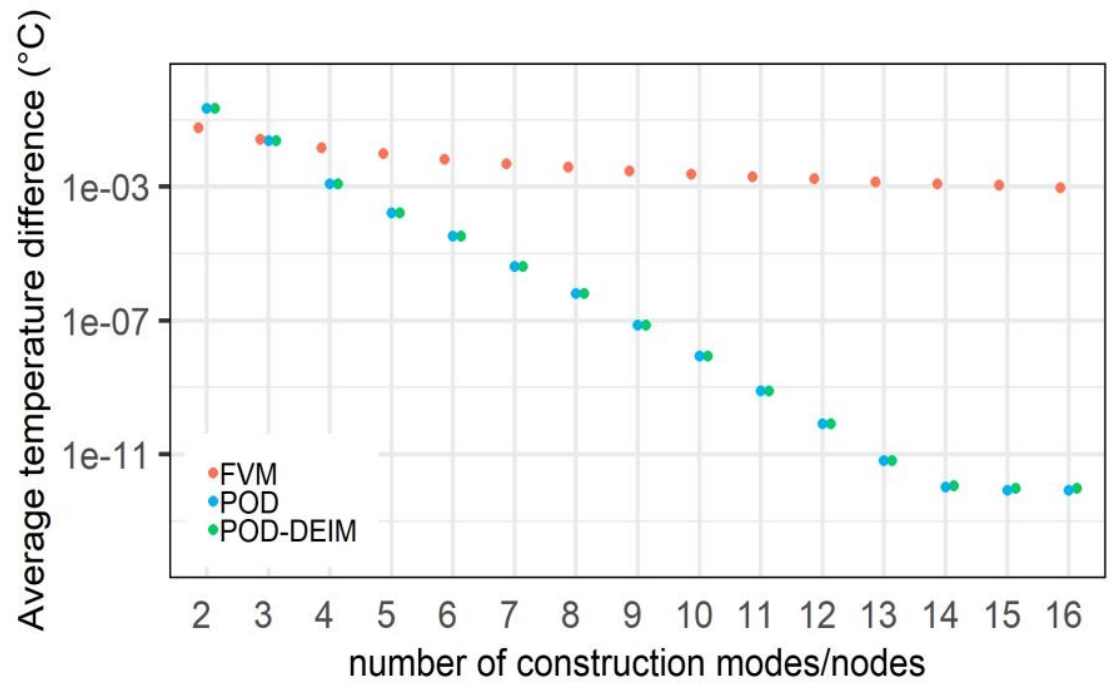

Figure 7: Average absolute temperature differences between the reference solution and solutions of the FVM, POD and POD-DEIM models constructed by different number of nodes/modes.

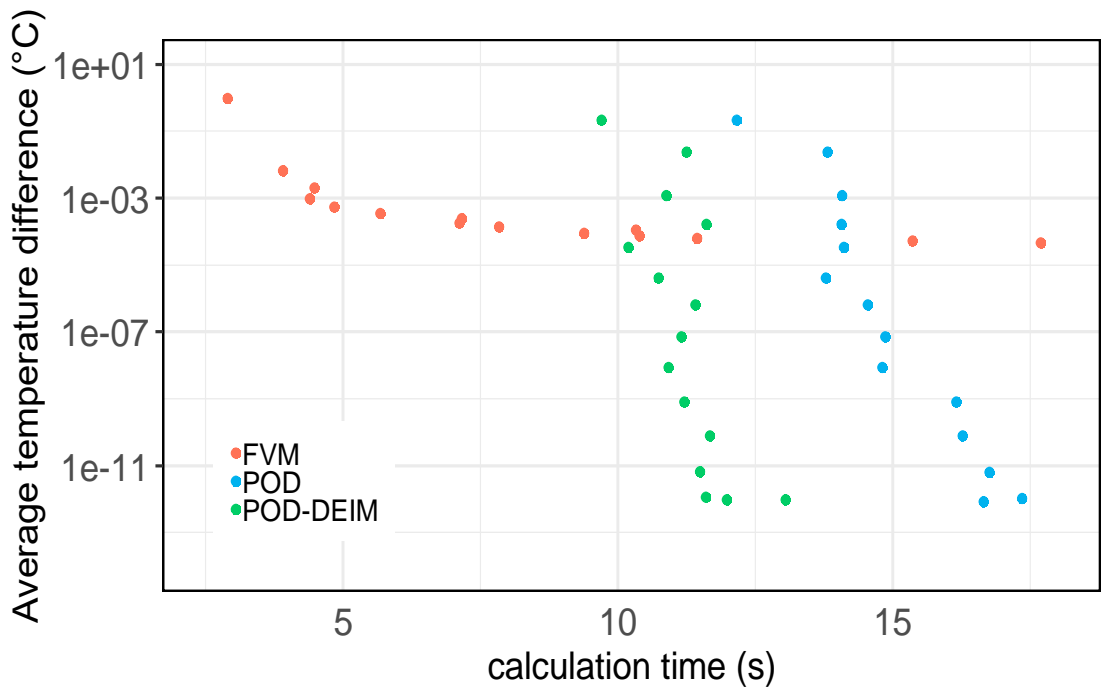

Figure 8: Average absolute temperature differences between the reference solution and solutions of the FVM, POD and POD-DEIM models, as a function of the calculation time.

It is demonstrated in Figure 4 that the singular values of the snapshots related to both solutions and nonlinear terms decrease very rapidly. The low plateaus of these two singular value curves appear after the $16^{\text {th }}$ and $26^{\text {th }}$ singular value respectively. In addition, the differences between the first and the $16^{\text {th }}$ singular values of the snapshot related to solutions and the first and the $26^{\text {th }}$ singular values of the snapshot related to nonlinear terms are around $10^{14}$ and $10^{16}$, respectively. These results reveal a strong interrelation of the snapshots related to both solutions and nonlinear terms. This suggests that rather small numbers of POD modes and DEIM points suffice for accurate results of the POD-DEIM. 
Figure 5 and 6 demonstrate that there are no perceivable differences between the reference solution, the POD and POD-DEIM model with 16 modes, and the FV model with 16 nodes, for temperature profiles. In other words, all the three models can provide relatively accurate profiles of the temperature distributions. On the other hand, larger differences are found for the solutions calculated by the FVM, POD and POD-DEIM models with only 2 nodes or modes. These outcomes confirm that, with a sufficient number of nodes or modes, all three methods can provide accurate results.

With respect to the temperature deviations, Figure 7 reveals that the average absolute temperature differences between the reference and the FVM, POD and POD-DEIM models decrease as the number of their nodes/modes raise. It is demonstrated that the POD and POD-DEIM accuracy improves much faster than FVM though, and with more than 4 modes/nodes, POD and PODDEIM (strongly) outperforms FVM. On the other hand, it also shows with the same amount of modes, the accuracies of POD and POD-DEIM are very identical.

Figure 8 finally confronts the obtained calculation accuracies with the required computation times. This demonstrates that at limited accuracies (average absolute temperature differences above $10^{-4}$ ${ }^{\circ} \mathrm{C}$ ) the FVM is the most efficient. At higher accuracies (average absolute temperature differences below $10^{-5}{ }^{\circ} \mathrm{C}$ ) on the other hand, POD-DEIM is obviously the best method. Comparing with FVM, POD-DEIM allows to obtain a much better accuracy at a given computation time. For the same level of accuracy, the calculation cost of POD-DEIM tends to be much smaller than the POD method.

\subsubsection{POD-DEIM for longer simulation intervals}

In section 3.1.1, it was shown that the POD-DEIM model based on the full set of solution snapshots can provide a very accurate result, and that it strongly outperforms FVM and POD when a highly accurate result is desired. However, in practice this recalculation of the same problem does not add any value, as all the information is already included in the solution snapshots. Therefore, the use of POD-DEIM for simulating problems with longer simulation periods is investigated in this section. To do so, several POD-DEIM models are constructed by using the same input values mentioned in section 3.1.1, but with solution snapshots constructed from shorter simulation time periods: one month, one day, half day, six hours, three hours. These shorter simulation periods are taken at the start of the year. The resulting reduced models are then executed to simulate the hourly temperature profiles over an entire year. To give a detailed illustration of the POD-DEIM performance with solution snapshots from different simulation periods, the average absolute temperature differences between the reference solution and POD-DEIM models with the solution snapshots taken from different shorter simulation periods, as a function of the number of POD modes are shown in Figure 9. Here all the POD-DEIM models are constructed by 35 DEIM points. It should be mentioned that, the reduced models constructed by the snapshots of shorter simulation periods (also by different simulation configurations) have the same structure as those constructed by the large original model. As a result, the computational cost of the reduced models constructed by the simulations of shorter periods and of the large original model are equal. Therefore, there is no need to compare their performance in terms of computational time saving, as all the results are already illustrated in Figure 8. 


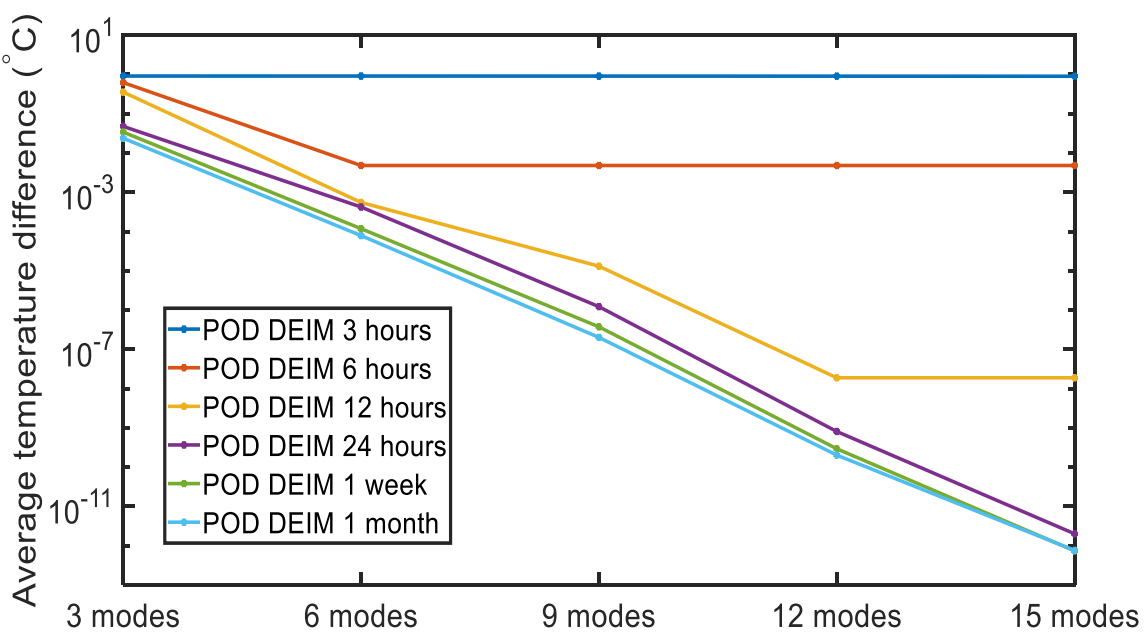

Figure 9: Average temperature differences between reference solution and POD-DEIM models constructed by the solution snapshots of different simulation periods.

Figure 9 illustrates that, except for POD-DEIM models constructed from 3 hours and 6 hours of solution snapshots, the average temperature differences between the reference solution and the POD-DEIM models decrease significantly as the number of their POD modes raise. Similar as Figure 7, Figure 9 also shows that with a sufficient number of modes, the performance of PODDEIM for simulating problems with longer simulation periods is very satisfactory. Only when the number of snapshots is really insufficient (from simulation periods of three or six hours), an inaccurate result may be obtained. This implies that very limited earlier simulation results, covering only a single full or half day of actual standard calculation, suffice to reproduce the longterm dynamic temperature behavior of the building component (the average temperature difference may go below $10^{-11}$, when a high number of modes is employed). This was already demonstrated for the linear case in [9], but is now extended to nonlinear conditions here.

\subsubsection{POD-DEIM for different simulation configurations}

Beside using POD-DEIM for simulating problems with longer simulation periods, another advantage of POD-DEIM is that one can execute the reduced models for simulating problems with different boundary conditions and/or material properties. In this section we investigate the performance of POD-DEIM when simulating problems with configurations different from the training solution snapshots. More specifically, 5 scenarios are considered. In the first 4 scenarios, selected separate parameters are modified relative to the original, an overview of which is collected in Table 1 . For the $5^{\text {th }}$ scenario, all modifications of these 4 scenarios are imposed simultaneously. It should be noted that the differences between the training and the testing properties are designed to be relatively large, to cover a wide spectrum of configurations, and hence the training properties in Table 1 differ from the configuration applied in section 3.1.1. In addition, like in section 3.1.2, all POD-DEIM models are constructed based on snapshots of one single day (the first day of the year), and the resulting reduced models are then executed to simulate the hourly temperature profiles over an entire year. The average absolute temperature differences between the reference solution and the POD-DEIM models in relation to the 5 scenarios are shown in Figure 10. Here all the POD-DEIM models are constructed by 35 DEIM points. 
Table 1: Training properties and testing properties of the 5 scenarios

\begin{tabular}{llll}
\hline Scenario & Element modified & Training properties & Testing properties \\
\hline 1 & Material properties & $\rho: 2100 \mathrm{~kg} / \mathrm{m}^{3} \& \lambda: 1 \mathrm{~W} / \mathrm{mK}$ & $\rho: 1700 \mathrm{~kg} / \mathrm{m}^{3} \& \lambda: 0.6 \mathrm{~W} / \mathrm{mK}$ \\
2 & Transfer coefficients & $h_{i}: 6 \mathrm{~W} / \mathrm{m}^{2} \mathrm{~K} \& h_{e}: 10 \mathrm{~W} / \mathrm{m}^{2} \mathrm{~K}$ & $h_{i}: 10 \mathrm{~W} / \mathrm{m}^{2} \mathrm{~K} \& h_{e}: 40 \mathrm{~W} / \mathrm{m}^{2} \mathrm{~K}$ \\
3 & Interior temperature & $T_{\text {interior }}: 15^{\circ} \mathrm{C}$ & $T_{\text {interior }}: 25^{\circ} \mathrm{C}$ \\
4 & Exterior temperature & $T_{\text {exterior }}:$ Roma (Italy) & $T_{\text {exterior }}$ Arjeplog (Sweden) \\
5 & All modifications of the above 4 scenarios are imposed simultaneously \\
\hline
\end{tabular}

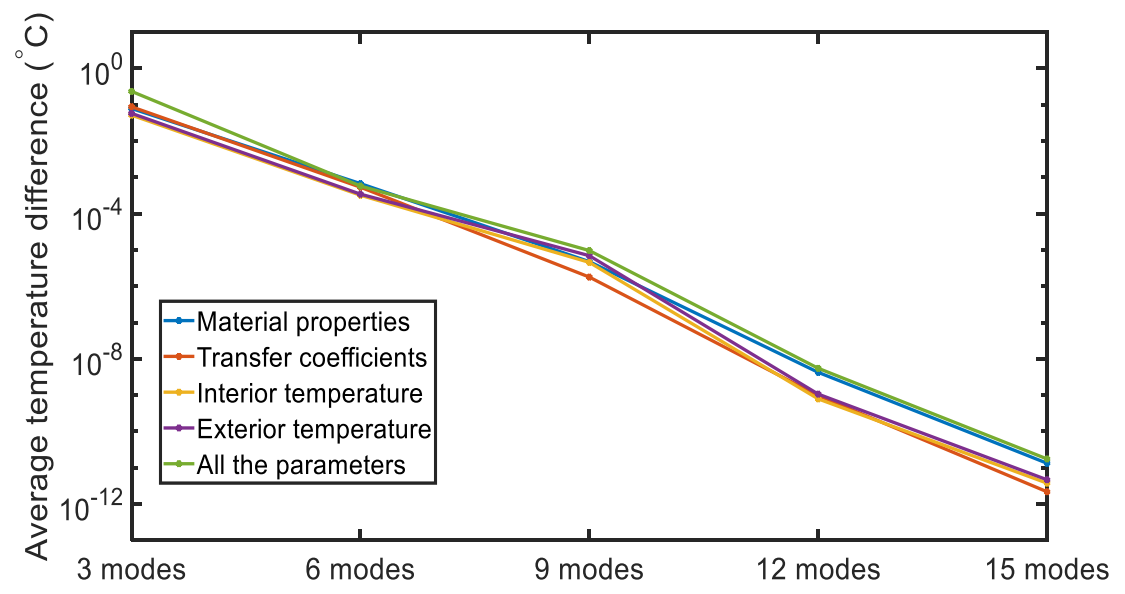

Figure 10: Average absolute temperature differences between reference solution and the POD-DEIM models constructed with different boundary conditions and material properties.

Figure 10 again illustrates that the accuracy of POD-DEIM, when simulating with different configurations, increases as the number of their modes raises. As a result, it is illustrated that in all the 5 scenarios, with a sufficient number of construction modes, the POD-DEIM method can provide an accurate result. This confirms the final observation of the section above, indicating that very few earlier simulation results, covering a single full day of actual standard calculation on a single configuration, suffice to quantify the long-term dynamic temperature behavior of a spectrum of building component configurations.

\subsection{Scenario 2: temperature-dependent thermal conductivity}

This case study is similar to the case study shown in section 3.1, but now the source of nonlinearity comes from the temperature-dependent thermal conductivity. All the models are constructed by using the same input values mentioned in section 3.1.1, except for thermal capacity and thermal conductivity. In this example, the thermal capacity is assumed constant: $870 \mathrm{~J} / \mathrm{kgK}$. The value of the thermal conductivity $\lambda$ is $0.5 \mathrm{~W} / \mathrm{mK}$ below $0^{\circ} \mathrm{C}$ and $1.5 \mathrm{~W} / \mathrm{mK}$ above $30^{\circ} \mathrm{C}$, with a linear dependence on temperature between these two bounds.

In this case study, only the performance of FVM, POD and POD-DEIM constructed by the full set of snapshots is investigated, as these results contain the most important findings. More specifically, the analysis in Figure 4 and Figure 8 are re-performed, but now the nonlinear source changes from 
thermal capacity to thermal conductivity. The results are shown in Figure 11 and 12 respectively. Figure 11 presents again the decay of the first 45 singular values of the snapshots related to both reference solution and nonlinear terms. In Figure 12, the average temperature differences are illustrated as a function of the calculation time. Here, the POD models are constructed by 2 to 16 modes, and for each POD model 35 DEIM points are used to build the POD-DEIM models. To bring the results of all the three methods in a comparable range, the FV models are constructed by 35 to 105 nodes, with an interval of 5 nodes.

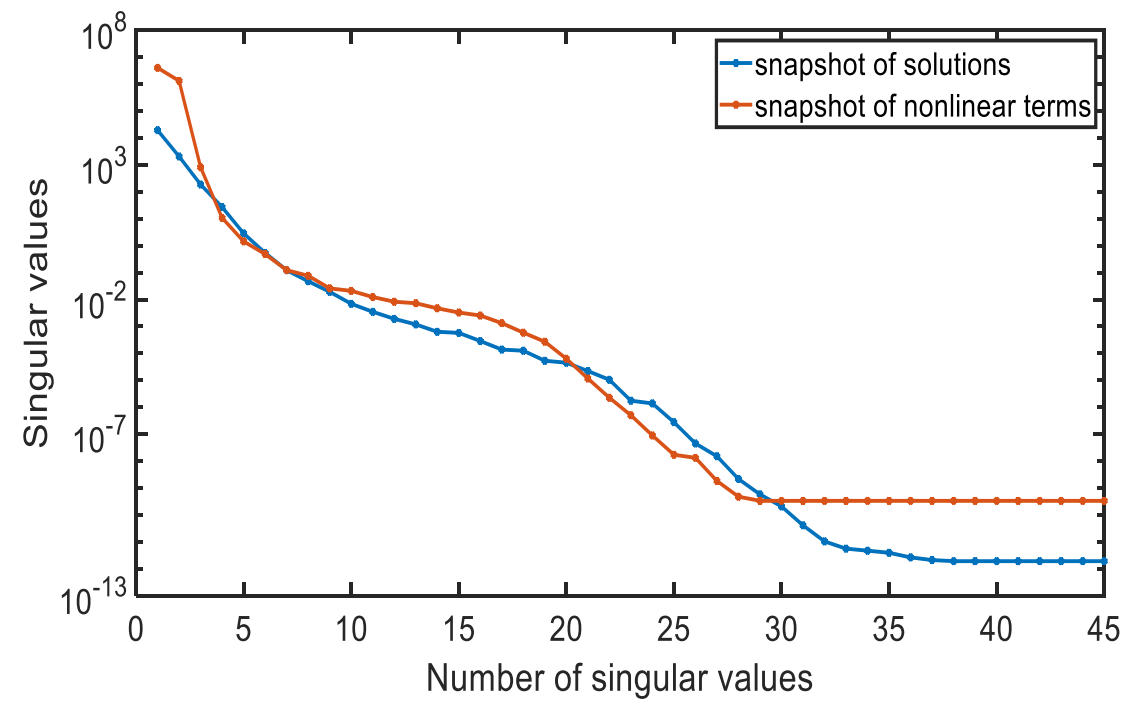

Figure 11: The decay progress of the first 45 singular values of the snapshots related to both the reference solution and the nonlinear terms.

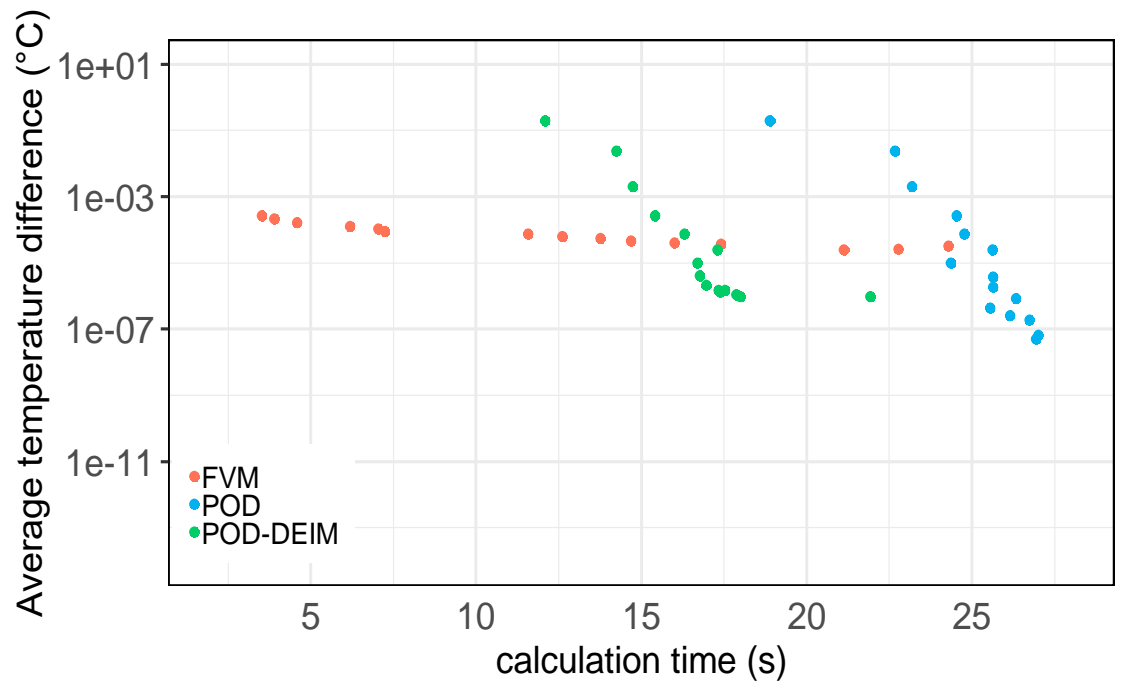

Figure 12: Average absolute temperature differences between the reference solution and solutions of the POD, POD-DEIM and FV models, as a function of the calculation time.

Figure 11 shows that the singular values of the snapshots related to both solutions and nonlinear terms decrease as the their numbers increase. Comparing with Figure 4, the plateaus of the two 
singular value curves appear after the $29^{\text {th }}$ and $32^{\text {nd }}$ singular values, respectively. In addition, the differences between the first and the $16^{\text {th }}$ singular values of the solutions snapshot and the first and the $26^{\text {th }}$ singular values of nonlinear terms snapshot are around $10^{9}$ and $10^{11}$, respectively. Therefore, it is demonstrated that the singular value decays of the snapshots in Figure 11 are slower than the singular value decays of the snapshots in Figure 4. These findings suggest that the snapshots shown in Figure 11 have weaker interrelations than the snapshots shown in Figure 4. These results provide support for the view that more POD modes and DEIM points are required to build a POD-DEIM model for the simulation of heat transfer problems with nonlinear thermal conductivity than with nonlinear thermal capacity.

Figure 12 confirms the findings of Figure 11. With the same number of the POD modes, the temperature errors of POD and POD-DEIM can only reach around $10^{-7}{ }^{\circ} \mathrm{C}$. Comparing with Figure 8 , where the minimal temperature errors of POD and POD-DEIM are around $10^{-12}{ }^{\circ} \mathrm{C}$, we again conclude that the use of POD and POD-DEIM for quantifying the heat transfer with nonlinear thermal conductivity requires more POD modes than quantifying the heat transfer with nonlinear thermal capacity. In addition, comparing the calculation time of Figure 8 and Figure 12, it is also shown that with the same number of construction modes, using POD and POD-DEIM for quantifying the heat transfer with nonlinear thermal conductivity requires more calculation time (12s to $27 \mathrm{~s})$ than for quantifying the heat transfer with nonlinear thermal capacity ( $7 \mathrm{~s}$ to $17.5 \mathrm{~s})$.

The lower calculation time required for the simulation of scenario 1 than scenario 2 can be explained in terms of two aspects. First, for all the numerical models used in this paper (FVM, POD, POD-DEIM), the thermal capacity and thermal conductivity are respectively used to construct the mass and stiffness matrices. In addition, in relation to the FVM, the mass and stiffness matrices are respectively in the format of diagonal and tridiagonal matrices. Since the FVM serves as the basis for POD and POD-DEIM, the mass and stiffness matrices of POD and POD-DEIM are also preserved with the same formats. Equation (5) and (11) respectively illustrated that, for both POD and POD-DEIM, in each iteration the mass and stiffness matrices need to be multiplied with the POD basis, and the complexity of the both cases are $k n+n^{2}$ and $3 k n+n^{2}$, respectively. Therefore, the first case requires less operations than the second case and hence less calculation time. Second, in the FVM when the source of nonlinearity comes from the thermal conductivity, the thermal conductivity averages between all the neighboring volumes need to be calculated to assess the heat transport between the volumes. Hence, in the framework of POD-DEIM, not only the thermal conductivities of the selected volumes, but also the thermal conductivities of their neighboring volumes need to be evaluated. On the other hand, for the case with nonlinear thermal capacity, these calculations of the averages between all the neighboring volumes are not required. As a result, for the POD-DEIM, only the thermal capacities of the selected volumes need to be evaluated, leading to a lower computational cost.

\section{Case study 2: nonlinear moisture transfer}

In section 3, it is shown that when a highly accurate result is desired, POD-DEIM is the most efficient method, which (strongly) outperforms FVM and POD for nonlinear heat transfer through a building material. In this section, a more complex isothermal hygric case study on moisture transfer in a building material caused by sudden drops of the relative humidity at both surfaces, is performed. More specifically, HAMSTAD benchmark 2 [22] is selected as the example, since it 
has been widely used as a reference benchmark to assess the performance of different simulation models [23]-[25]. A material layer of $0.2 \mathrm{~m}$ thick at high initial relative humidity (95\%) is suddenly exposed to low surface relative humidities ( $45 \%$ on exterior side and $65 \%$ on the interior side), which causes a drying process which is simulated over an interval of 100 hours. Detailed information about HAMSTAD benchmark 2 -construction geometry, material properties, boundary conditions - can be found in [22]. The case study is simulated here respectively by FVM, POD and POD-DEIM, and all solvers necessary for the purpose have been developed by the first author. The accuracy of the obtained solutions is then evaluated by comparison with a reference solution from a FVM calculation with 200 spatial mesh elements and a fixed time step of one hour, and the simulation duration is 100 hours. A sketch of the material with boundary conditions is illustrated in Figure 13.

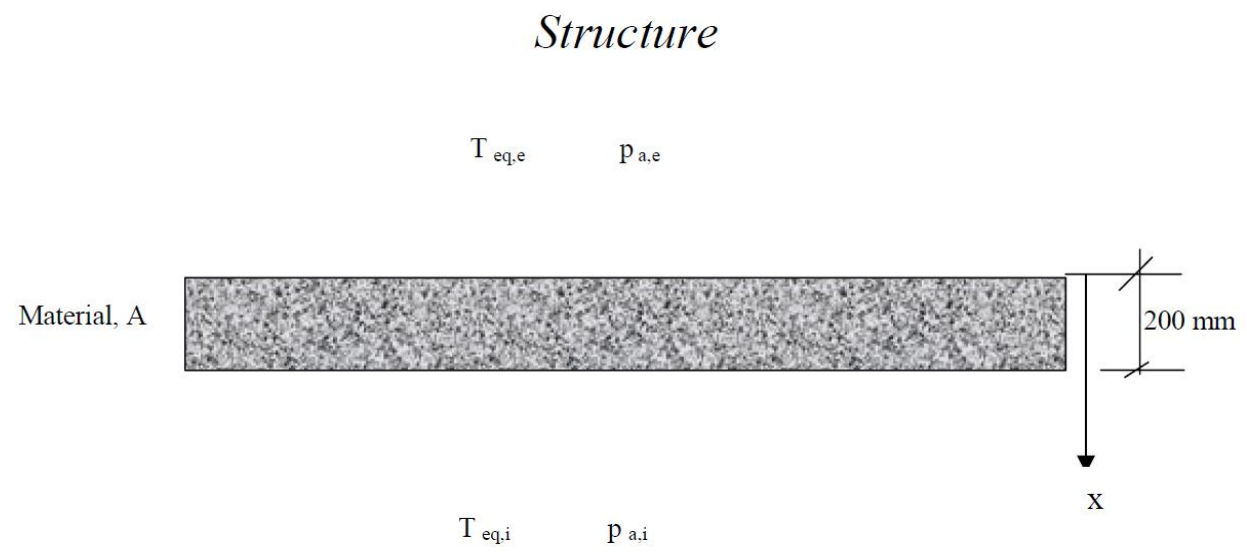

Figure 13: A sketch of the material with boundary conditions of the moisture transfer case study. (from [22])

In this section, for the sake of simplicity and without lost of generality, two research issues are addressed: 1. the accuracy and computational cost of FVM, POD and POD-DEIM constructed with the full set of snapshots are compared. 2. the performance of POD and POD-DEIM for simulating problems with longer simulation intervals is studied. The results are respectively put forward in sections 4.1 and 4.2. In order to compare the overall behavior of different models, the deviation of solutions of different models relative to the reference solution are quantified with the average absolute moisture content difference. The average absolute moisture content difference is calculated similarly as the average absolute temperature difference, see equation (14), but replaces the temperatures by moisture contents. 
Postprint: Hou T., Meerbergen K., Roels S., Janssen H., 2020. POD-DEIM model order reduction for nonlinear heat and moisture transfer in building materials, Journal of Building Performance Simulation, 13:6, 645-661, DOI: 10.1080/19401493.2020.1810322

\subsection{FVM, POD, POD-DEIM constructed by the full set of snapshots}

This section is a direct copy of section 3.1.1, but now for modelling nonlinear moisture transfer. The calculation accuracy and computational expense of FVM and POD and POD-DEIM constructed by the full set of snapshots are judged. First, to illustrate the decay speed of the singular values of the shanpshots related to both the reference solution and the nonlinear terms, the decay progress of the first 50 singular values of these two snapshots are respectively shown in Figure 14. Next, for getting a more direct view of the performance of different models, we select both the spatial moisture content profiles at 100 hours and the temporal evolution of the moisture content at $0.19 \mathrm{~m}$ of the building component as target outputs. The results are respectively presented in Figures 15 and 16. The case study is simulated by the three models with different number of modes/nodes. More specifically, the FVM models are constructed by 5 and 50 nodes. The POD models are constructed by 5 and 10 modes, and for each POD model 50 DEIM points are used to build the POD-DEIM models. For the FVM, in order to obtain moisture content values at the spatial points of the reference solution, the intermediate values are calculated by interpolation. Further, to obtain a comprehensive view of the performance of different models, the average absolute moisture content deviations of different models are compared, shown in Figure 17. More specifically, the FVM and POD models are constructed by 5, 10, 15, 20, 25 nodes/modes. For each POD model, different number of DEIM points (65 points, 55 points, 45 points, 35 points, 25 points) are used to build the POD-DEIM models. Finally, as the number of modes/nodes and DEIM points do not entirely represent the computational expense of different models, the average moisture content differences are again presented in Figure 18, but now as a function of the calculation time, to provide a fair comparison of the computational performance of different approaches. Here, all the models are used to predict the moisture content profiles for 100 hours. To bring the results of all the three methods in a comparable range, the FVM models are constructed with 80 to 150 nodes, with an interval of 5 nodes. The POD models are constructed with 4 to 18 modes. In relation to the POD-DEIM models, 7, 10 and 25 POD modes are used together with 40 to 44 DEIM points.

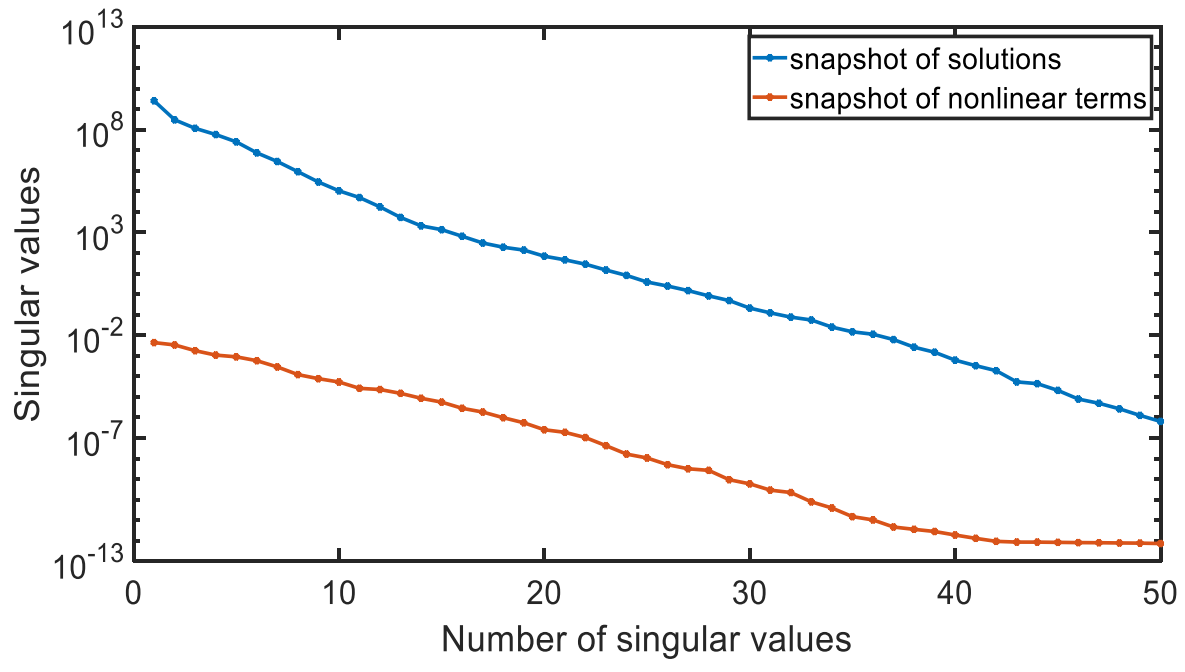


Figure 14: The decay progress of the first 50 singular values of the snapshots related to both the reference solution and the nonlinear terms.

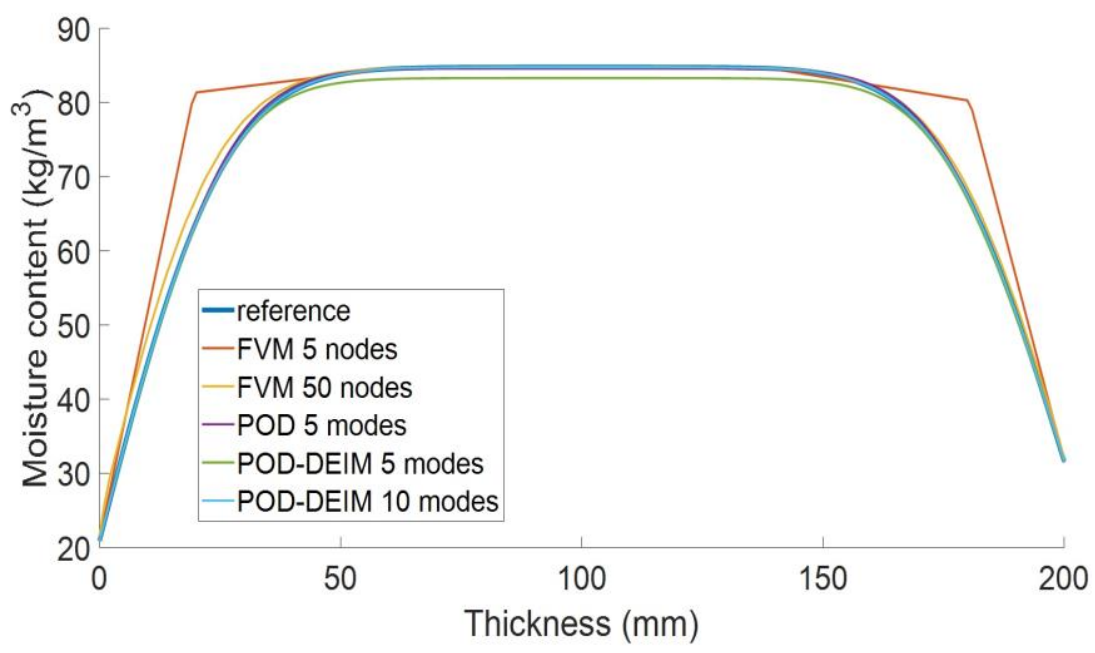

Figure 15. Spatial moisture content profiles at 100 hours, for the reference solution and solutions of FVM models and reduced models constructed by different number of nodes/modes; coordinate ' 0 m' refers to the top surface.

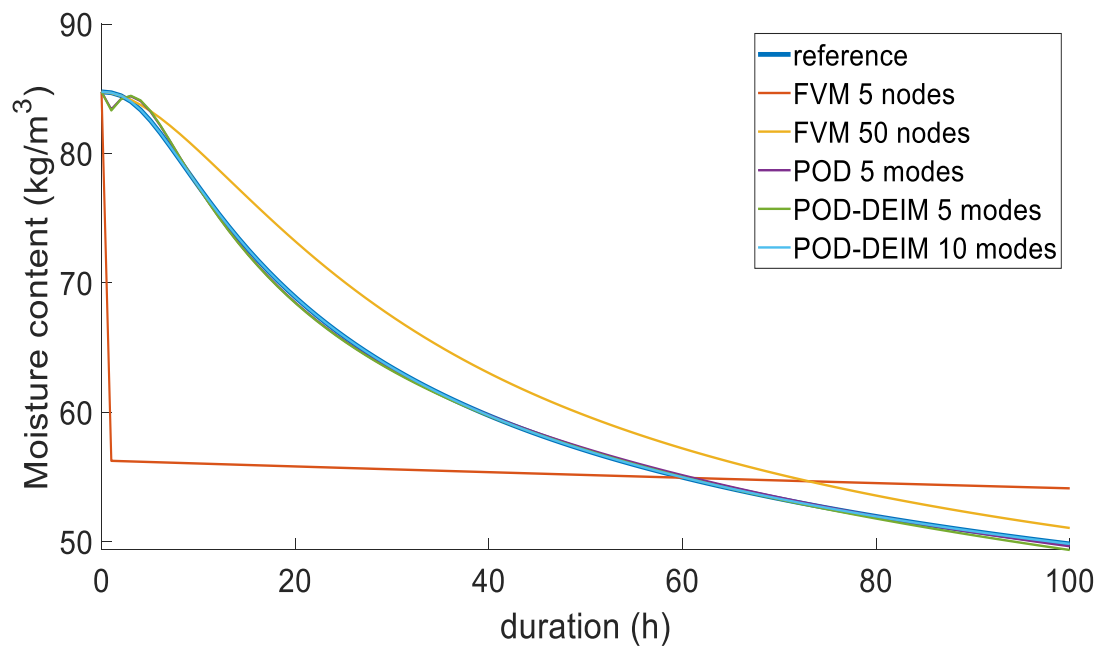

Figure 16. Temporal evolution of moisture content at $0.19 \mathrm{~m}$ of the building component of the reference solution and solutions of FVM models and reduced models constructed by different number of nodes/modes; duration ' 0 h' refers to the start of the simulation. 


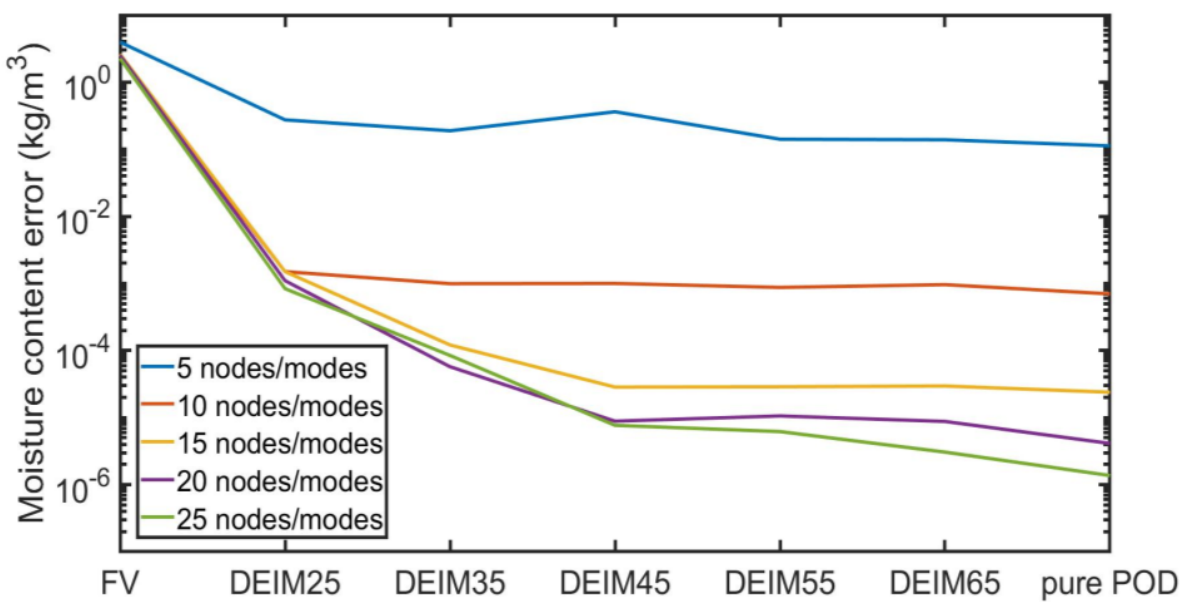

Figure 17. Average absolute moisture content difference between the reference solution and solutions of FVM models and reduced models constructed by different number of nodes/modes and DEIM points.

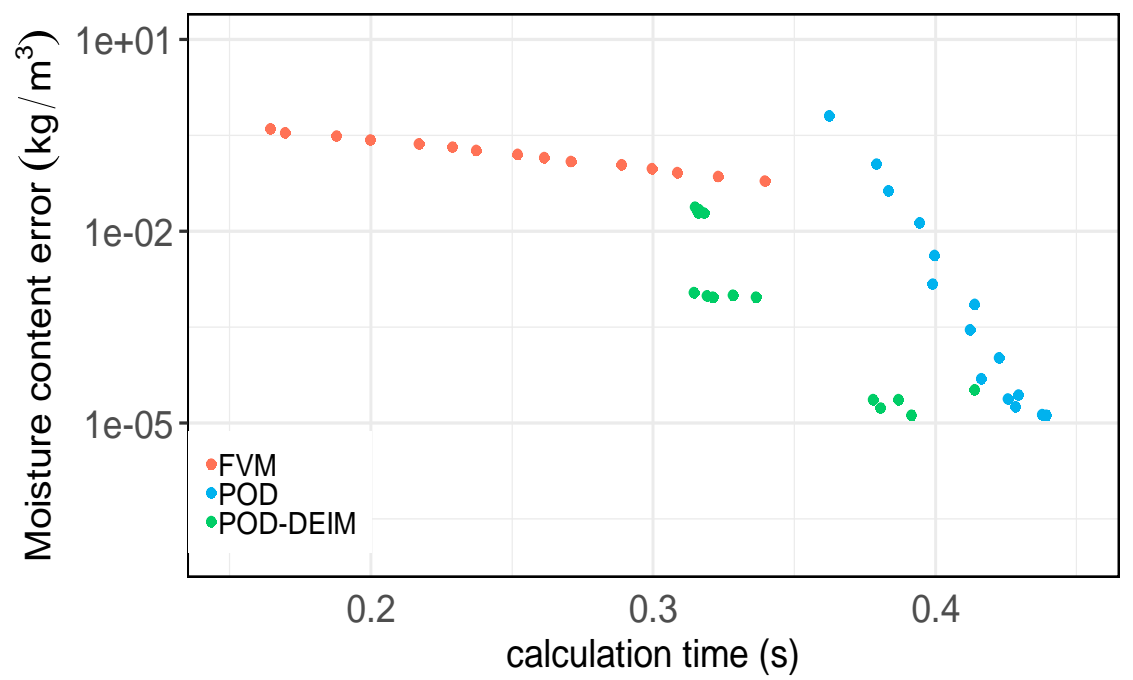

Figure 18. Average absolute moisture content differences between the reference solution and solutions of the FVM, POD and POD-DEIM models, as a function of the calculation time.

Figure 14 shows that, the singular values of the snapshots related to both solution and nonlinear terms decrease as their numbers increase. Different from Figure 4 and 11, we do not see an obvious plateau for the singular value curve of the solution snapshot. On the other hand, the plateau for the singular value curve of nonlinear terms snapshot appears after the $40^{\text {th }}$ singular value. Comparing with Figure 4 and 11, the decay speeds of the both singular value curves in Figure 14 tend to be (much) slower. These results suggest that the snapshots shown in Figure 14 have smaller interrelations than the snapshots shown in Figure 4 and 11. Therefore, comparing with the use of POD-DEIM for the simulation of heat transfer problems, more POD modes and DEIM points are demanded for a POD-DEIM model to simulate the nonlinear moisture transport problems. 
Figure 15 and 16 show that for the POD model with 5 modes and POD-DEIM model with 10 modes there is no observable differences between the reference solution and the reduced models' approximations. Hence, for HAMSTAD benchmark 2, with a relatively small number of construction modes and DEIM points, both POD and POD-DEIM can provide an accurate result of the moisture distribution in the material layer. On the other hand, a rather large differences can be found between the FVM model with only 5 nodes and the reference solution.

Figure 17 presents an overview of the accuracies of different models constructed by different number of POD modes and DEIM points. It confirms the results of Figure 15 and 16, with a sufficient number of construction modes and interpolation points, POD-DEIM can provide an accurate result and the accuracy of the POD-DEIM increases as the amount of their construction modes and DEIM points raises. On the other hand, Figure 17 also shows that with more than 45 DEIM points the accuracies of the reduced models do not increase significantly. This result confirms the observations in Figure 14 that the singular values of the nonlinear terms snapshot do not decrease significantly after the $45^{\text {th }}$ singular value.

Figure 18 finally confronts the obtained calculation accuracies with the required computation times. This demonstrates that at large inaccuracies (moisture content errors around $0.1 \mathrm{~kg} / \mathrm{m}^{3}$ ) the FVM is the most efficient. At better accuracies (moisture content errors below $0.01 \mathrm{~kg} / \mathrm{m}^{3}$ ) on the other hand, POD-DEIM is obviously the most efficient method, since it allows to obtain the best accuracy at a given computation time, strongly outperforming FVM and POD. It should be mentioned that, in this paper all the POD and POD-DEIM models are based on the snapshots of the reference solution, and hence the deviation of solutions of POD and POD-DEIM relative to the reference solution tends to get very small. We also investigated the performance of POD and PODDEIM models that are derived from simulation deviating from the reference solution. As the results hereof are similar to Figure 18, they are not explicitly presented in the paper.

\subsection{POD-DEIM for longer simulation intervals}

Similar as section 3.1.2, this section investigates the use of POD-DEIM to construct a reduced model for simulating different problems in relation to longer simulation period. In other words, the accuracies of the POD-DEIM models constructed by different size of snapshots are evaluated. More specifically, for getting a more direct view of the performance of POD-DEIM as a function of the size of its snapshot, similar as before the moisture content profiles of the building component simulated by POD and POD-DEIM constructed by 18 modes and different size of snapshots are shown in Figure 19. Here, all the POD-DEIM models are built with 50 DEIM points. In addition, to further evaluate the accuracy of POD and POD-DEIM methods as a function of the amount of the construction modes, the average moisture content differences between the reference solution, POD and POD-DEIM models built by different size of snapshots, as a function of the amount of their POD modes are shown in Figure 20. Similar as Figure 19, all the POD-DEIM models are built with 50 DEIM points. 


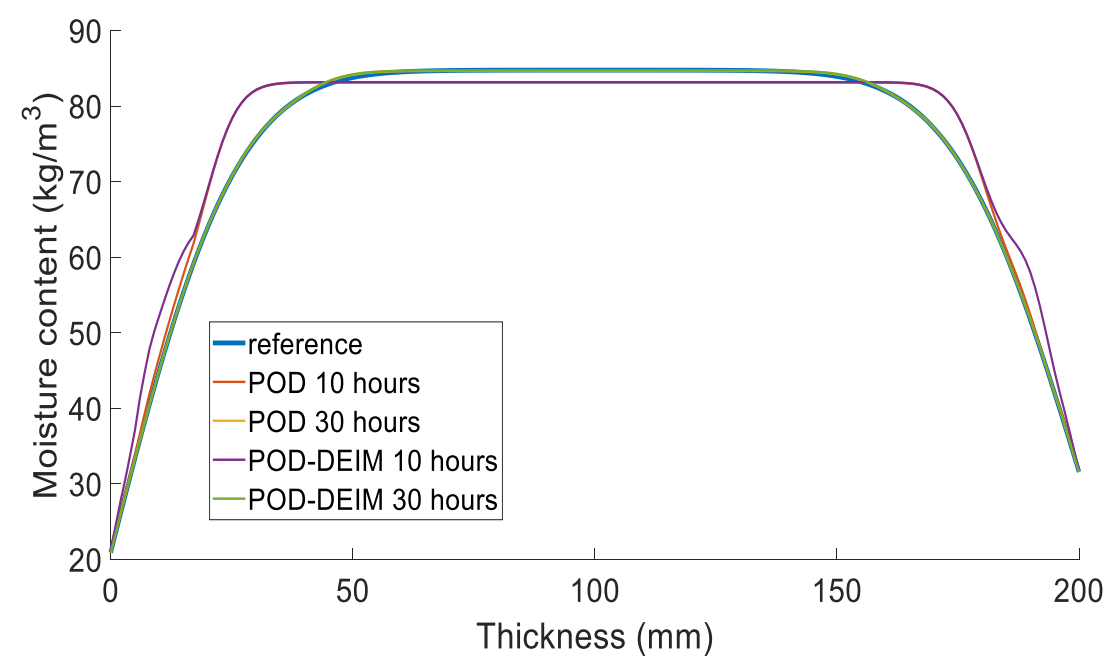

Figure 19. Moisture content profiles at $\mathbf{1 0 0}$ hours of the reference solution and solutions of the reduced models constructed by different size of snapshot.

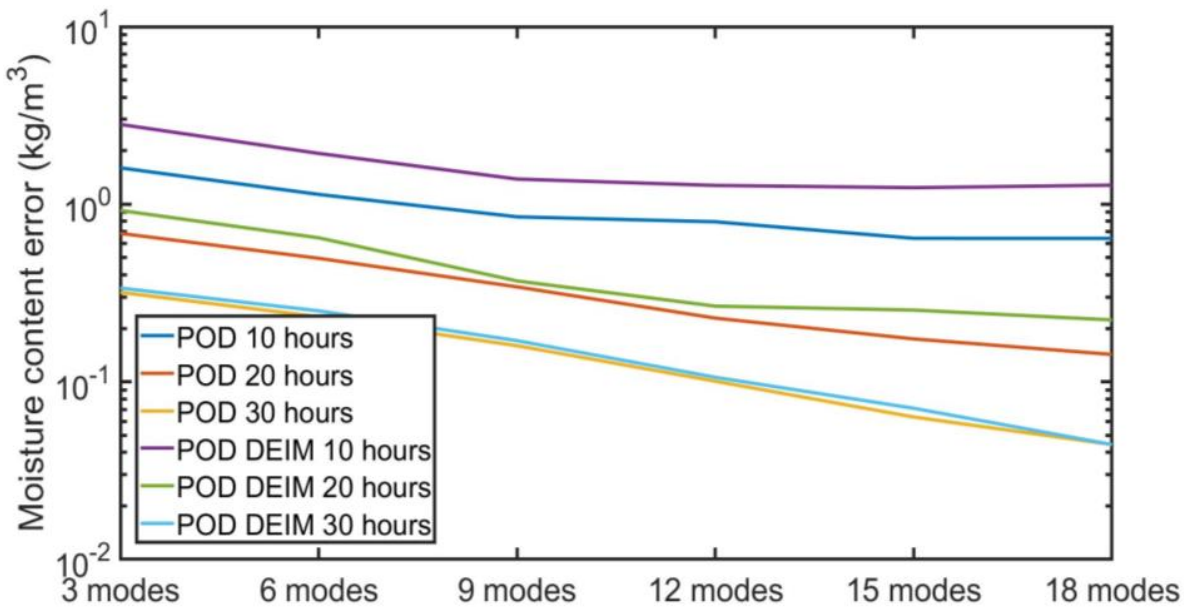

Figure 20. Average absolute moisture content difference between the reference solution and solutions of the reduced models constructed by different size of snapshots and different number of construction modes.

Figure 19 is similar to Figure 15, but now for different size of snapshots. It is demonstrated that, with 30 hours snapshot and 18 modes, the moisture distribution in the material layer can be obtained rather accurately by both POD and POD-DEIM models. On the other hand, larger differences can be found between the reduced models with only 10 hours snapshot and the reference solution.

Figure 20 again illustrates that the accuracies of POD and POD-DEIM increase as the size of their snapshots raises. It is also shown that, with a sufficient size of snapshot (from simulation intervals of 30 hours), the accuracies of the POD and POD-DEIM models increase as the number of their modes raises. On the other hand, when the size of snapshots is insufficient (from simulation of 10 hours), an inaccurate result (moisture content errors larger than $1 \mathrm{~kg} / \mathrm{m}^{3}$ ) may be obtained. As result, this confirms the final observation of the section above, indicating that with a 30 hours of 
actual standard calculation, suffice to reproduce the long-term moisture content behavior of the building component. However, comparing with the heat transfer problem in section 3 , a larger size of snapshot is required for capturing the moisture content profile than the temperature distribution.

\section{Conclusion}

In this paper, the potential use of POD-DEIM for modelling nonlinear heat and moisture transport problems are assessed via two applications: a simple case study of nonlinear heat transfer through a building component and a more complexed case study about analyzing the isothermal moisture redistribution in one building material layer. In both of the two applications it is shown that PODDEIM model has the ability to be more efficient than the FVM and POD, and is capable to deliver a reliable result for simulating problems deviate from the snapshot solution. Although comparing with POD these computational time savings are only in the range of a few seconds (Fig. 8 and Fig. 12), for the probabilistic analysis where many repetitions of deterministic simulations are required [26], the total accumulated computational cost reduction can be huge. On the other hand, we also found that the intensity of the nonlinearity influences the performance of POD-DEIM. In addition, similar as the POD method, the performance of POD-DEIM is highly determined by the interrelation of the snapshots related to both the solution and nonlinear terms. Normally, the higher interrelation of the snapshots, the less POD modes and DEIM points are required, and hence lead to a larger reduction of the calculation cost.

This paper is a first attempt to investigate the feasibility of POD-DEIM for nonlinear problems in building physics and both examples in section 3 and 4 are still rather theoretical and simple case studies. Further research is needed to check whether this good performance can also be achieved for the more complicated and strongly non-linear (coupled) heat and moisture transport problems typically simulated by building component models.

\section{Acknowledgement}

This work was supported by the European Union's Horizon 2020 research and innovation program under grant agreement No 637268 (RIBuild).

\section{References}

[1] J. Grunewald, "Diffusiver und konvektiver Stoff-und Energie-transport in kapillarporösen Baustoffen."

[2] H. M. Künzel, "Verfahren zur ein-und zweidimensionalen Berechnung des gekoppelten Wärme-und Feuchtetransports in Bauteilen mit einfachen Kennwerten," Universität Stuttgart Stuttgart, 1994.

[3] T. D. Pettersen, "Variation of energy consumption in dwellings due to climate, building and inhabitants," Energy Build., vol. 21, no. 3, pp. 209-218, 1994.

[4] H. Janssen, B. Blocken, and J. Carmeliet, "Conservative modelling of the moisture and heat transfer in building components under atmospheric excitation," Int. J. Heat Mass Transf., 
vol. 50, no. 5, pp. 1128-1140, 2007.

[5] M. Jadidi, F. Bazdidi-Tehrani, and M. Kiamansouri, "Scale-adaptive simulation of unsteady flow and dispersion around a model building: spectral and POD analyses," J. Build. Perform. Simul., vol. 11, no. 2, pp. 241-260, 2018.

[6] K. Li, X. Wenping, C. Xu, and H. Mao, “A multiple model approach for predictive control of indoor thermal environment with high resolution," J. Build. Perform. Simul., vol. 11, no. 2, pp. 164-178, 2018.

[7] W. Liu, M. Jin, C. Chen, and Q. Chen, "Optimization of air supply location, size, and parameters in enclosed environments using a computational fluid dynamics-based adjoint method," J. Build. Perform. Simul., vol. 9, no. 2, pp. 149-161, 2016.

[8] A. Tallet, E. Liberge, and C. Inard, "Fast POD method to evaluate infiltration heat recovery in building walls," in Building Simulation, 2017, vol. 10, no. 1, pp. 111-121.

[9] T. Hou, S. Roels, and H. Janssen, "A comparison of model order reduction methods for the simulation of wall heat transfer," Proc. 7th Int. Build. Phys. Conf., pp. 1295--1300, 2018.

[10] S. Chaturantabut and D. C. Sorensen, "Nonlinear model reduction via discrete empirical interpolation,” SIAM J. Sci. Comput., vol. 32, no. 5, pp. 2737-2764, 2010.

[11] P. Tiso and D. J. Rixen, "Discrete empirical interpolation method for finite element structural dynamics," in Topics in Nonlinear Dynamics, Volume 1, Springer, 2013, pp. 203212.

[12] I. . Ştefănescu, R. and Navon, "POD/DEIM nonlinear model order reduction of an ADI implicit shallow water equations model," J. Comput. Phys., vol. 237, pp. 95-114, 2013.

[13] S. Chaturantabut and D. C. Sorensen, "Application of POD and DEIM on dimension reduction of non-linear miscible viscous fingering in porous media," Math. Comput. Model. Dyn. Syst., vol. 17, no. 4, pp. 337-353, 2011.

[14] I. . Ştefănescu, R., Sandu, A. and Navon, "POD/DEIM reduced-order strategies for efficient four dimensional variational data assimilation," J. Comput. Phys., vol. 295, pp. 569-595, 2015.

[15] H. Yang and A. Veneziani, "Efficient estimation of cardiac conductivities via POD-DEIM model order reduction," Appl. Numer. Math., vol. 115, pp. 180-199, 2017.

[16] D. D. Kosambi, "Statistics in function space," in DD Kosambi, Springer, 2016, pp. 115123.

[17] Y. C. Liang, H. P. Lee, S. P. Lim, W. Z. Lin, K. H. Lee, and C. G. Wu, "Proper orthogonal decomposition and its applications-Part I: Theory," J. Sound Vib., vol. 252, no. 3, pp. 527544, 2002. 
[18] C. Sumiyoshi et al., "Semantic Memory Organization in Japanese Patients With Schizophrenia Examined With Category Fluency," Front. psychiatry, vol. 9, p. 87, 2018.

[19] A. Fic, R. A. Białecki, and A. J. Kassab, "Solving transient nonlinear heat conduction problems by proper orthogonal decomposition and the finite-element method," Numer. Heat Transf. Part B Fundam., vol. 48, no. 2, pp. 103-124, 2005.

[20] D. Pasetto, A. Guadagnini, and M. Putti, "POD-based Monte Carlo approach for the solution of regional scale groundwater flow driven by randomly distributed recharge," Adv. Water Resour., vol. 34, no. 11, pp. 1450-1463, 2011.

[21] R. Pinnau, "Model reduction via proper orthogonal decomposition," in Model order reduction: theory, research aspects and applications, Springer, 2008, pp. 95-109.

[22] C.-E. Hagentoft, "Hamstad wp2-benchmark package," 2002.

[23] A. W. M. van Van Schijndel, "Heat and Moisture Modeling Benchmark using COMSOL," in Proceedings of the COMSOL Users Conference 2008 Hannover, 2008.

[24] Q. Li, J. Rao, and P. Fazio, "Development of HAM tool for building envelope analysis," Build. Environ., vol. 44, no. 5, pp. 1065-1073, 2009.

[25] X. Liu, Y. Chen, H. Ge, P. Fazio, and G. Chen, "Numerical investigation for thermal performance of exterior walls of residential buildings with moisture transfer in hot summer and cold winter zone of China," Energy Build., vol. 93, pp. 259-268, 2015.

[26] T. Hou, D. Nuyens, S. Roels, and H. Janssen, "Quasi-Monte Carlo based uncertainty analysis: Sampling efficiency and error estimation in engineering applications," Reliab. Eng. Syst. Saf., vol. 191, p. 106549, 2019. 